\title{
MULTIDISCIPLINARY INTERVENTION FOR SELF-MANEGEMENT OF PAIN IN FIBROMYLAGIA: A MIXED METHOD STUDY
}

Patricia Arenas Rocha ${ }^{1}$, Larissa Hiromi Sayama Esteves ${ }^{1}$, Callebe Frizzas ${ }^{1}$, Mariane Louise Bonato ${ }^{2}$, Lais Zanlorenzi ${ }^{3}$, Bárbara Stadler Kahlow ${ }^{1,3, \star}$, Eduardo dos Santos Paiva ${ }^{2}$, Patricia Martin ${ }^{1,3}$

1.Pontifícia Universidade Católica do Paraná, Curitiba (PR), Brazil.; 2.Universidade Federal do Paraná, Curitiba (PR), Brazil.; 3.Hospital Universitário Evangélico Mackenzie, Curitiba (PR), Brazil.

*Corresponding author: bazinha_st@yahoo.com.br

\section{BACKGROUND}

Fibromyalgia (FM) is a chronic disease that results in an important compromise in quality of life, with no single effective treatment. Therefore, in addition to medications, it demands multidisciplinary care that includes understanding the disease and activities aimed at physical and psychological well-being. In this way, the aim of the present study was to evaluate a group multidisciplinary intervention for self-management of chronic pain in patients with FM in a public health secondary attention service.

\section{METHODS}

The intervention consisted of 10 weekly meetings offered to groups of 4 to 15 FM patients. Psychoeducation, cognitive behavior therapy, mindfulness, breathing exercises and muscle relaxation, neuromuscular positional release for pain relief, guidance on joint protection, organization of routines, diet and sleep hygiene were performed by a multidisciplinary team. To assess the program's impact, visual analogue scale (VAS) for pain, fatigue and sleep quality and revised fibromyalgia impact questionnaire (FIQr), applied before, at the end of treatment and after one year, were used. Qualitative analysis through focus groups using the methodology of content analysis according to Bardin were done after one year of follow-up. Because multiple comparison was done, Bonferroni correction was used and $p$ was considered significant if $\leq 0.025$. The size of the Cohen effect was calculated and considered small if between 0.1 and 0.29 ; moderate if between 0.3 and 0.5 and large when greater than 0.5. Research Ethics Committee Approval No. 2.063.758,05/15/17.

\section{RESULTS}

At the end of the intervention, functional capacity evaluated by FIQr ( $p=0.002$, size effect $=0.88)$, fatigue $(p=0.006$, size effect $=0.59)$ and sleep quality $(p=0.017$, size effect $=0.53)$ improved and, after one year of follow up, sleep quality $(p=0.003$, size effect $=0.66)$ and fatigue $(p=0.023$, size effect $=0.519)$ improvement were maintained. Content analysis demonstrated that better acceptance of themselves and their disease, organization in daily routine, use of energy conservation, measures and practice of relaxation exercises were observed in the group. The participants requested the maintenance of the meetings.

\section{CONCLUSION}

The group multidisciplinary intervention for self-management of chronic pain was effective in improving fatigue and sleep quality at short and long term and also to improve functional capacity at short term. Indeed, qualitative analysis demonstrated that these results could be a consequence of better acceptance of themselves and their disease, organization in daily routine, use of energy conservation measures and practice of relaxation exercises.

\section{KEYWORDS}

Fibromyalgia, Self-management, Therapy group intervention, Multidisciplinary approach. 